\title{
Association of Time to First Morning Cigarette and Chronic Obstructive Pulmonary Disease Measured by Spirometry in Current Smokers
}

\author{
Geonhyeok Kim', Hongji Song' ${ }^{1}$ Kyunghee Park', Hyemi Noh' ${ }^{1}$ Eunyoung Lee', Hyoeun Lee' ${ }^{1}$, Hayoon Kim², Yujin Paek ${ }^{1, *}$ \\ 'Department of Family Medicine, Hallym University Sacred Heart Hospital, Anyang, Korea \\ ${ }^{2}$ Department of Family Medicine, Hallym University Kangnam Sacred Heart Hospital, Seoul, Korea
}

Background: Time to first cigarette after waking is an indicator of nicotine dependence. We aimed to identify the association between time to first cigarette and spirometry-proven obstructive respiratory impairment, especially chronic obstructive pulmonary disease, in current smokers.

Methods: We included 392 subjects who visited the comprehensive medical examination center of Hallym University Sacred Heart Hospital between July 2014 and September 2015. Subjects with lung disease or anemia were excluded. Obstructive pulmonary impairment was defined as $<70 \%$ of the predicted value of forced expiratory volume in 1 second/forced vital capacity. Subjects were classified into the early ( $\leq 30$ minutes) and late ( $>30$ minutes) groups based on the time to first cigarette. Logistic regression and linear regression analyses were used for data analysis.

Results: Ninety-eight subjects (25\%) were classified into the early group. After adjusting for smoking behaviors (cigarettes per day and smoking duration), socioeconomic status (education and income), age, and physical activity, an early time to first cigarette was found to be associated with an increased risk of obstructive pulmonary impairment measured using spirometry (adjusted odds ratio, 2.84; 95\% confidence interval, 1.22-6.61).

Conclusion: Compared to current smokers with a late time to first cigarette, those with an early time to first cigarette had a higher risk of obstructive pulmonary impairment, especially chronic obstructive pulmonary disease. Classifying smoking-related behaviors, especially time to first cigarette, may help target clinical screening for chronic obstructive pulmonary disease.

Keywords: Smoking; Chronic Obstructive Pulmonary Disease; Time to First Cigarette; Nicotine Dependence; Respiratory Function Tests

Received: June 29, 2016, Revised: October 17, 2016, Accepted: October 20, 2016

*Corresponding Author: Yujin Paek https://orcid.org/0000-0001-9573-8849

Tel: +82-31-380-3805, Fax: +82-31-380-1782, E-mail: paek@hallym.or.kr 


\section{INTRODUCTION}

Chronic obstructive pulmonary disease (COPD) is a respiratory disease characterized by an irreversible limitation in airflow. ${ }^{1)}$ The high prevalence and mortality rate of COPD has imposed a significant economic burden on both patients and health care delivery systems. ${ }^{2)}$ According to the World Health Organization statistics, there were 210 million people with COPD worldwide in 2000, and it was the fifth leading cause of death in 2002. ${ }^{3)}$ The fifth Korea National Health and Nutrition Examination Survey, conducted between 2010 and 2012 in South Korea, showed that the prevalence of COPD increased during each of the 3 years of the study period, with a mean prevalence of $13.7 \%$ among those surveyed. ${ }^{4-6)}$ Furthermore, lung diseases, including COPD, were the seventh leading cause of death in Korea. ${ }^{4-6)}$

The risk factors of COPD include smoking, airway hyperresponsiveness, occupational exposures, and genetic diseases such as $\alpha_{1}$ antitrypsin deficiency. ${ }^{1)}$ Among these factors, smoking is estimated to be the primary causal factor for $80 \%$ of COPD-related deaths. ${ }^{7)}$ Smoking behavior and addiction can be analyzed using the Fagerstrom Test for Nicotine Dependence (FTND) that takes into account the time to first cigarette of the day (TTFC). The TTFC is the first item in the FTND and is known to reflect nicotine addiction and the ability to quit smoking. ${ }^{8)}$ A recent study on the relationship between COPD and TTFC concluded that a shorter TTFC correlates with an increased risk of COPD. ${ }^{9)}$ However, COPD was self-reported by the subjects in that study, and neither that nor other studies have used pulmonary function testing (PFT). PFT is an important measure for diagnosing obstructive lung disease, but clinical guidelines advise against screening asymptomatic patients for COPD owing to the associated costs. ${ }^{1,10)}$ Consequently, asymptomatic smokers can be easily overlooked. However, a single question regarding a smoker's TTFC would be cost-effective if shown to be a good screening method for smokers at risk of COPD. ${ }^{9)}$

Therefore, this study evaluated the relationship between TTFC and obstructive pulmonary disease, especially COPD, as measured by using spirometry in current smokers who visited Hallym University Sacred Heart Hospital.

\section{METHODS}

\section{Subjects}

This study was a cross-sectional analysis. For medical examination, 3,142 adults over 20 years of age (men: 1,930; women: 1,212) visited the Hallym University Sacred Heart Hospital between July 2014 and September 2015. Among them, we included 437 self-reported current smokers in the study. We excluded subjects with a self-reported history of lung disease such as asthma, tuberculosis, COPD, pneumothorax, and anemia because these conditions could affect the results of PFT $(17,4,2,2$, and 1 patient, respectively). Additionally, we excluded current female smokers and subjects whose PFT showed restrictive respiratory impairment because of their small sample numbers (12 and 7 patients, respectively). Finally, 392 subjects were included in the pres- ent study.

All subjects signed informed consent forms before participating in this study. All study protocols were approved by the institutional review board of the Hallym University Sacred Heart Hospital (IRB approval no., 2014-I013).

\section{Pulmonary Function Testing}

PFT is typically performed using spirometry. ${ }^{1)}$ In this study, PFT was performed by trained technicians using a digital spirometer (Viasys Respiratory Care Inc., Palm Springs, CA, USA). Before the test, subjects were directed to avoid several things that could affect the PFT results, as recommended by the American Thoracic Society and European Respiratory Society. For example, subjects were told to avoid smoking and drinking alcohol for 24 hours before the test. During the test, subjects were seated and a nose clip was used to prevent air from escaping from the nose.

PFT is an effort-dependent test used to assess for obstructive pulmonary impairment. ${ }^{1)}$ Participants were instructed to inhale deeply and blow into a mouthpiece attached to the spirometer as hard and fast as they could. We recorded the forced vital capacity (FVC), forced expiratory volume in 1 second (FEV1), and FEV1/FVC. Obstructive pulmonary impairment was defined as $<70 \%$ of the predicted value of FEV1/ FVC.

\section{Clinical Variables}

We collected the following information about the subjects by using a structured questionnaire: TTFC, cigarette smoking behavior, alcohol consumption, physical activity, income, and education. Based on their responses to a questionnaire regarding TTFC, the subjects were categorized into one of the following four groups: within 5 minutes of waking, 6-30 minutes after waking, 31-60 minutes after waking, or >60 minutes after waking. Because of the small number of subjects, the use of four separate categorizations of TTFC was ineffective for analyzing the association between TTFC and obstructive pulmonary impairment (Appendix 1). Therefore, the subjects were subsequently classified into the early ( $\leq 30$ minutes after waking) and late ( $>30$ minutes after waking) TTFC groups. For smoking behavior analysis, we collected subject data and categorized subjects into different groups depending on smoking duration and cigarettes per day (CPD). Participants who consumed >14 alcoholic drinks per week were indicated as "heavy drinkers." ${ }^{\text {11) }}$ We classified subjects who performed at least 150 minutes of moderate-intensity aerobic physical activity throughout the week as the exercise group. ${ }^{12)}$ Weight and height were measured by trained nurses using electronic equipment. Body mass index (BMI) was calculated as weight $(\mathrm{kg}) / \mathrm{height}(\mathrm{m})^{2}$ and subjects were classified as obese $\left(\geq 25 \mathrm{~kg} / \mathrm{m}^{2}\right)$ or not obese $\left(<25 \mathrm{~kg} / \mathrm{m}^{2}\right)$. To determine the socioeconomic status (SES), we used two variables: income and education. Subjects were classified as having an income $<400$ Korean million won or $\geq 400$ Korean million won. ${ }^{13)}$ Education level was based on the attainment of a high school degree or a bachelor's degree. 


\section{Statistical Analysis}

In the present study, we analyzed all data by using IBM SPSS Statistics for Windows ver. 22.0 (IBM Corp., Armonk, NY, USA). Using the Kolmogorov-Smirnov test, categorical and continuous variables were assessed for normality. The $\chi^{2}$ test was used to analyze the relationship between TTFC and several variables, and Student t-test was used to analyze categorical variables and continuous variables. Bivariate and multivariate logistic regression analyses were conducted to evaluate the risk of an obstructive ventilatory disturbance according to TTFC. Bivariate and multivariate linear regression analyses were conducted to evaluate the relationship between FVC\%, FEV1\%, and FEV1/FVC according to TTFC. We performed bivariate (unadjusted; model 1) analysis of the relationship between early TTFC and obstructive pulmonary impairment, FEV1, FVC, and FEV1/FVC (Tables 1, 2). The associations between early TTFC and obstructive pulmonary impairment, FEV1, FVC, and FEV1/FVC were also analyzed after adjusting for smoking behaviors (CPD and smoking duration; model 2) and in a fully adjusted model (smoking behaviors [CPD and smoking duration], SES [education and income], age, and physical activity; model 3). In these models, we also included physical activity, which was not significant in the univariate analysis (Table 3), but could affect lung function and have a significant association with nicotine dependence. ${ }^{14,15)}$ All tests were two sided, and the level of significance was set at $\mathrm{P}<0.05$.

Table 1. Bivariate or multiple logistic regression analysis of the risk of obstructive ventilatory disturbance group according to TTFC

\begin{tabular}{|c|c|c|c|}
\hline \multirow{2}{*}{ Model } & \multirow{2}{*}{ Predictor } & \multicolumn{2}{|c|}{ Outcome (obstructive pulmonary impairment) } \\
\hline & & Odds ratio ( $95 \%$ confidence interval) & P-value \\
\hline \multirow[t]{2}{*}{ Model $1^{*}$} & TTFC $\leq 30 \mathrm{~min}$ & $2.72(1.29-5.74)$ & 0.009 \\
\hline & TTFC $>30 \mathrm{~min}$ & 1 & \\
\hline \multirow[t]{2}{*}{ Model $2^{\dagger}$} & TTFC $\leq 30 \mathrm{~min}$ & $2.90(1.37-6.17)$ & 0.006 \\
\hline & TTFC $>30 \mathrm{~min}$ & 1 & \\
\hline \multirow[t]{2}{*}{ Model $3^{\ddagger}$} & TTFC $\leq 30 \mathrm{~min}$ & $2.84(1.22-6.61)$ & 0.016 \\
\hline & TTFC $>30 \mathrm{~min}$ & 1 & \\
\hline
\end{tabular}

TTFC, time to first cigarette; CPD, cigarettes per day.

*Unadjusted (bivariate logistic regression and linear regression analyses). ${ }^{\dagger}$ Adjusted

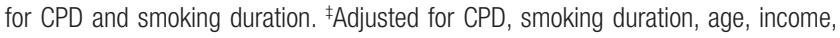
education, and physical activity.

\section{RESULTS}

The baseline characteristics of the participants are shown in detail in Table 3. In this study, we divided the subjects into two groups according to TTFC (early TTFC: $\leq 30$ minutes after waking; late TTFC: $>30$ minutes after waking). About $25 \%$ of the current smokers were classi-

Table 3. Characteristics of subjects in the early TTFC ( $\leq 30 \mathrm{~min})$ and late TTFC ( $>30$ min) groups

\begin{tabular}{|c|c|c|c|}
\hline Characteristic & $\begin{array}{c}\text { Late TTFC } \\
>30 \min (n=294)\end{array}$ & $\begin{array}{c}\text { Early TTFC } \\
\leq 30 \text { min }(n=98)\end{array}$ & $P$-value \\
\hline Pulmonary function & & & 0.007 \\
\hline Normal pulmonary function & $277(94)$ & $84(86)$ & \\
\hline Obstructive pulmonary impairment & $17(6)$ & $14(14)$ & \\
\hline FVC (\%) & $98.99 \pm 11.61$ & $98.44 \pm 10.89$ & 0.669 \\
\hline FEV1 (\%) & $102.18 \pm 11.99$ & $100.86 \pm 12.04$ & 0.343 \\
\hline FEV1/FVC & $0.79 \pm 6.58$ & $0.77 \pm 7.34$ & 0.010 \\
\hline Age (y) & $44.66 \pm 7.16$ & $47.15 \pm 8.33$ & 0.002 \\
\hline Height (cm) & $172.69 \pm 6.35$ & $170.69 \pm 5.60$ & 0.003 \\
\hline Weight (kg) & $73.05 \pm 10.38$ & $70.94 \pm 9.74$ & 0.062 \\
\hline Body fat percentage & $22.41 \pm 5.44$ & $22.77 \pm 5.82$ & 0.556 \\
\hline Body mass index $\left(\mathrm{kg} / \mathrm{m}^{2}\right)$ & & & 0.875 \\
\hline$<25$ & $190(57)$ & $62(57)$ & \\
\hline$\geq 25$ & $146(44)$ & $46(43)$ & \\
\hline Cigarettes per day & & & 0.000 \\
\hline$\leq 20$ & $222(67)$ & $52(47)$ & \\
\hline$>20$ & $112(34)$ & $58(53)$ & \\
\hline Smoking duration (y) & & & 0.019 \\
\hline$<20$ & $93(28)$ & $18(17)$ & \\
\hline$\geq 20$ & $243(72)$ & $91(84)$ & \\
\hline Education & & & 0.007 \\
\hline High school degree & $127(38)$ & $57(53)$ & \\
\hline Bachelor's degree & $207(62)$ & $51(47)$ & \\
\hline Income (Korean million won) & & & 0.272 \\
\hline$<4(\$ 3,535)$ & $119(36)$ & $44(42)$ & \\
\hline$\geq 4(\$ 3,535)$ & $21(64)$ & $61(58)$ & \\
\hline Heavy drinking & $173(57)$ & $54(53)$ & 0.445 \\
\hline Physical activity & $84(26)$ & $33(32)$ & 0.226 \\
\hline
\end{tabular}

Values are presented as mean \pm standard deviation or number (\%). P-values were calculated using t-tests or $\chi^{2}$ tests.

TTFC, time to first cigarette; FVC, forced vital capacity; FEV1, forced expiratory volume in 1 second.

Table 2. Bivariate or multivariate linear regression analysis of FVC\%, FEV1\%, and FEV1/FVC according to TTFC

\begin{tabular}{|c|c|c|c|c|c|c|c|}
\hline \multirow{3}{*}{ Model } & \multirow{3}{*}{ Predictor } & \multicolumn{6}{|c|}{ Outcome } \\
\hline & & \multicolumn{2}{|c|}{ FVC\% } & \multicolumn{2}{|c|}{ FEV1\% } & \multicolumn{2}{|c|}{ FEV1/FVC } \\
\hline & & $\mathrm{B}(\mathrm{SE})$ & P-value & B (SE) & P-value & $\mathrm{B}(\mathrm{SE})$ & P-value \\
\hline \multirow[t]{2}{*}{ Model $1^{*}$} & $\mathrm{TTFC} \leq 30 \mathrm{~min}$ & $-0.551(1.286)$ & 0.669 & $-1.620(1.397)$ & 0.247 & $-1.907(1.790)$ & 0.016 \\
\hline & TTFC >30 min & 1 & & 1 & & 1 & \\
\hline \multirow[t]{2}{*}{ Model $2^{\dagger}$} & TTFC $\leq 30 \mathrm{~min}$ & $-0.87(1.311)$ & 0.947 & $-1.498(1.432)$ & 0.296 & $-1.189(0.778)$ & 0.128 \\
\hline & TTFC >30 min & 1 & & 1 & & 1 & \\
\hline \multirow[t]{2}{*}{ Model $3^{\ddagger}$} & TTFC $\leq 30 \mathrm{~min}$ & $0.928(1.366)$ & 0.498 & $-2.116(1.502)$ & 0.16 & $-0.600(0.761)$ & 0.43 \\
\hline & TTFC >30 min & 1 & & 1 & & 1 & \\
\hline
\end{tabular}

FVC, forced vital capacity; FEV1, forced expiratory volume in 1 second; TTFC, time to first cigarette; B, unstandardized coefficient estimate; SE, standard error; CPD, cigarettes per day.

${ }^{*}$ Unadjusted (bivariate logistic regression and linear regression analyses). ${ }^{\dagger}$ Adjusted for CPD and smoking duration. ${ }^{\ddagger}$ Adjusted for CPD, smoking duration, age, income, education, and physical activity. 
fied into the early TTFC group. Their mean age was 45.3 years. Significant differences were found in obstructive pulmonary impairment, FEV1/FVC, age, height, smoking behaviors (CPD and smoking duration), and education between the early TTFC and late TTFC groups $(\mathrm{P}<0.05)$. Compared with the late TTFC group, the early TTFC group had a higher age and lower education level.

Table 1 shows the results of the bivariate and multivariate logistic regression analyses adjusted for smoking behaviors (CPD and smoking duration), SES (education and income), age, and physical activity. At the bivariate (unadjusted; model 1) level, early TTFC was significantly related to obstructive pulmonary impairment (odds ratio [OR], 2.72; 95\% confidence interval [CI], 1.29-5.74). The association between early TTFC and obstructive pulmonary impairment was similar in the model adjusted for smoking behaviors (CPD and smoking duration; model 2) and in the fully adjusted model (smoking behaviors [CPD and smoking duration], SES [education and income], age, and physical activity; model 3) at a statistically significant level (adjusted OR, 2.90; 95\% CI, 1.37-6.17 for model 2; adjusted OR, 2.84; 95\% CI, 1.226.61 for model 3 versus late TTFC). Table 2 shows the results of the bivariate and multivariate linear regression analysis of FVC\%, FEV1\%, and FEV1/FVC according to TTFC. TTFC was not significantly related to FVC\%, FEV1\%, and FEV1/FVC except at the bivariate level for FEV1/ FVC (unadjusted coefficient estimate, -1.907; standard error, 1.790). However, the frequent negative values for the unadjusted coefficient estimate indicated that early TTFC could be associated with decreased pulmonary function overall.

\section{DISCUSSION}

Among current smokers in the present study, early TTFC was significantly associated with a higher risk of obstructive pulmonary impairment. In addition, considering several factors such as disease prevalence, age, and smoking behaviors, obstructive pulmonary impairment can be regarded as COPD in the present study. ${ }^{1,4-6)}$ The relationship between TTFC and COPD was statistically significant after adjusting for several confounders. Several reasonable mechanisms might explain the relationship between early TTFC and a higher risk of COPD. For instance, early TTFC smokers are prone to take more and larger puffs; therefore, more nicotine may accumulate in early TTFC smokers than in late TTFC smokers, concomitantly leading to a higher dose of carcinogens present in tobacco. ${ }^{16)}$ In addition, the presence of some genes increases the risk for COPD and nicotine dependence. For example, single nucleotide polymorphisms of the 15q25 locus of the $\alpha$-nicotinic acetylcholine receptor (CHRNA 3/5) have been shown to simultaneously increase the risk of nicotine dependence and COPD, independent of smoking behaviors. ${ }^{17,18)}$ However, other genetic variations have been shown to be effected by smoking behaviors. For instance, CYP2A6, a nicotine-metabolism enzyme, is associated with both nicotine dependence ${ }^{19)}$ and $\mathrm{COPD}^{20)}$ and its activity is mediated by smoking behaviors. ${ }^{21)}$ Additional studies on the influence of genes and smoking behaviors on nicotine dependence and the development of COPD are necessary.

In the present study, some variables did not have a statistically significant association with TTFC. However, the CIs show the presence of similar trends between some variables and TTFC in the bivariate model. For example, just as nicotine dependence is associated with low SES, ${ }^{22)}$ in the present analysis, the early TTFC group had similar CI trends in association with SES parameters such as lower income and education levels, even though the association between income and early TTFC was not statistically significant $(\mathrm{P}=0.272)$. This may have occurred because our defined cutoff income values did not accurately reflect the different levels of SES. ${ }^{13)}$

Meanwhile, smoking and obesity are significant causes of mortality and morbidity worldwide. ${ }^{23)}$ However, the association between nicotine dependence and obesity has been controversial. Weight loss could be associated with nicotine dependence because of an increased metabolic rate and decreased caloric absorption resulting from a reduction in appetite. ${ }^{24)}$ However, heavy smokers may gain weight because they are more prone to adopt behaviors leading to weight gain than are nonsmokers or light smokers. Additionally, they consume fewer vegetables, adopt non-nutritious food intake, drink more alcohol, and have a tendency for less physical activity. ${ }^{25,26)}$ Our study also showed no significant association between TTFC and obesity (P-values: weight, 0.062; BMI, 0.875; and body fat percentage, 0.556). Therefore, further studies are needed to more precisely define the relationship between obesity and TTFC.

PFT is an important method for the diagnosis of COPD, but it has largely been excluded from previous cohort studies on the association between TTFC and COPD. ${ }^{9)}$ To our knowledge, the present study is the first to investigate the association between TTFC and PFT by using spirometry. Several reports have suggested an association between smoking behaviors and PFT. Greater addiction to nicotine in smokers is associated with a higher risk of obstructive lung disease. ${ }^{27)}$ Higher FTND scores reflect greater nicotine dependence among smokers with COPD than among smokers without $\mathrm{COPD}^{28)}$ and ex-smokers who quit smoking after a COPD diagnosis. ${ }^{27)}$ The relationship between smoking and poor PFT values was highly significant. Smokers had a 17.3-times greater risk of having impaired pulmonary functions than did nonsmokers. ${ }^{29)}$ The association between respiratory symptoms and smoking duration is statistically significant. ${ }^{30)}$ Significant negative relationships were observed between the duration and amount of smoking and PFT values. ${ }^{31)}$ In a similar vein, this study also showed that early TTFC was associated with obstructive pulmonary impairment by itself after adjusting for CPD and smoking duration (Table 1). Moreover, our model 3 (adjusted for smoking behaviors, age, education, income, and physical activity) revealed an association between early TTFC and obstructive pulmonary impairment. These data, including our findings, suggest that TTFC is a significant predictor of lung disease, especially COPD. Therefore, a single question regarding a smoker's TTFC may be a useful and cost-effective tool if it is verified to be an accurate screening method for smokers at risk of COPD. ${ }^{99}$ Further study of smoking-related behaviors, especially TTFC, may help 
direct clinical screening for COPD, a serious disease and public health burden.

This study has several limitations. COPD should be diagnosed clinically in any patient who has a postbronchodilator FEV1/FVC $<0.7$ by using spirometry. ${ }^{1)}$ Although the PFT data indicated obstructive respiratory impairment in the present study, it did not establish a diagnosis of COPD because we did not assess the response to bronchodilators in subjects with obstructive respiratory impairment. Therefore we could not exclude asthma, asthma-COPD overlap syndrome, bronchiectasis, and tuberculosis. ${ }^{1)}$ However, given the disease prevalence, age, and smoking behaviors, COPD was the most likely cause of obstructive pulmonary impairment in the present study. ${ }^{1,4-6)}$ The prevalence of obstructive pulmonary impairment in the present study was lower than the prevalence of COPD in Korea. This may be because the mean age of our subjects was about 45 years, and this relatively young age may have limited the degree of respiratory impairment caused by smoking. ${ }^{1)}$ In addition, subjects who came to the medical center for a checkup may have had a healthier lifestyle. We also excluded subjects with restrictive respiratory impairment on PFT because of their small number. Moreover, the association between TTFC, FEV1\%, and FVC\% did not show statistical significance in the univariate and bivariate analyses. In the present study, only nine subjects had an FEV1 below $80 \%$ (COPD stage $\geq 2$ ) (data not shown), and this may have affected the analysis of any association of TTFC with PFT values (FVC\%, FEV1\%, and FEV1/FVC).

Because of these reasons, additional larger studies, including PFTs with bronchodilators and TTFC, are needed to evaluate the relationship between lung function and nicotine dependence. Furthermore, large studies and cohort studies on patients of both sexes from a broader age range and varied ethnicities are needed because a greater understanding of smoking behaviors and COPD may allow earlier diagnosis and help develop improved strategies to limit disease progression.

\section{CONFLICT OF INTEREST}

No potential conflict of interest relevant to this article was reported.

\section{REFERENCES}

1. Global Strategy for the Diagnosis, Management and Prevention of COPD [Internet]. [place unknown]: Global Initiative for Chronic Obstructive Lung Disease (GOLD); 2017 [cited 2017 Dec 9]. Available from: http://goldcopd.org.

2. Park H, Jung SY, Lee K, Bae WK, Lee K, Han JS, et al. Prevalence of chronic obstructive lung disease in Korea using data from the fifth Korea national health and nutrition examination survey. Korean J Fam Med 2015;36:128-34.

3. World Health Organization. Global surveillance, prevention and control of chronic respiratory diseases [Internet]. Geneva: World Health Organization; 2007 [cited 2015 Nov 16]. Available from: http://apps. who.int/iris/bitstream.
4. Korea Centers for Disease Control and Prevention. Korean health statistics 2010: Korea National Health and Nutrition Examination Survey (KNHANES V-1). Cheongju: Korea Centers for Disease Control and Prevention; 2011.

5. Korea Centers for Disease Control and Prevention. Korean health statistics 2011: Korea National Health and Nutrition Examination Survey (KNHANES V-2). Cheongju: Korea Centers for Disease Control and Prevention; 2012.

6. Korea Centers for Disease Control and Prevention. Korean health statistics 2012: Korea National Health and Nutrition Examination Survey (KNHANES V-3). Cheongju: Korea Centers for Disease Control and Prevention; 2013.

7. Centers for Disease Control and Prevention. Smoking-attributable mortality, years of potential life lost, and productivity losses: United States, 2000-2004 [Internet]. Atlanta (GA): Centers for Disease Control and Prevention; 2008 [cited 2015 Nov16]. Available from: http://www. cdc.gov/.

8. Transdisciplinary Tobacco Use Research Center (TTURC) Tobacco Dependence, Baker TB, Piper ME, McCarthy DE, Bolt DM, Smith SS, et al. Time to first cigarette in the morning as an index of ability to quit smoking: implications for nicotine dependence. Nicotine Tob Res 2007;9 Suppl 4:S555-70.

9. Guertin KA, Gu F, Wacholder S, Freedman ND, Panagiotou OA, ReyesGuzman C, et al. Time to first morning cigarette and risk of chronic obstructive pulmonary disease: smokers in the PLCO Cancer Screening Trial. PLoS One 2015;10:e0125973.

10. Rostron BL, Chang CM, Pechacek TF. Estimation of cigarette smokingattributable morbidity in the United States. JAMA Intern Med 2014; 174:1922-8.

11. U.S. Department of Health and Human Services, U.S. Department of Agriculture. Dietary guidelines for Americans 2015-2020 eighth edition [Internet]. Rockville (MD): U.S. Department of Health and Human Services; 2015 [cited 2017 Dec 9]. Available from: http://health.gov/dietaryguidelines/2015/guidelines/.

12. World Health Organization. Global recommendations on physical activity for health [Internet]. Geneva: World Health Organization; 2010 [cited 2015 Nov16]. Available from: http://apps.who.int/iris/bitstream.

13. Hyundai Research Institute. Change on quality of life in the middle class in Korea [Internet]. Seoul: Hyundai Research Institute; 2015 [cited 2015 Nov16]. Available from: http://hri.co.kr.

14. Loprinzi PD, Walker JF. Nicotine dependence, physical activity, and sedentary behavior among adult smokers. N Am J Med Sci 2015;7:949.

15. Cheng YJ, Macera CA, Addy CL, Sy FS, Wieland D, Blair SN. Effects of physical activity on exercise tests and respiratory function. Br J Sports Med 2003;37:521-8.

16. Grainge MJ, Shahab L, Hammond D, O'Connor RJ, McNeill A. First cigarette on waking and time of day as predictors of puffing behaviour in UK adult smokers. Drug Alcohol Depend 2009;101:191-5.

17. Siedlinski M, Tingley D, Lipman PJ, Cho MH, Litonjua AA, Sparrow D, et al. Dissecting direct and indirect genetic effects on chronic obstructive pulmonary disease (COPD) susceptibility. Hum Genet 2013;132: 431-41.

18. Wilk JB, Chen TH, Gottlieb DJ, Walter RE, Nagle MW, Brandler BJ, et al. A genome-wide association study of pulmonary function measures in 
the Framingham Heart Study. PLoS Genet 2009;5:e1000429.

19. O'Loughlin J, Paradis G, Kim W, DiFranza J, Meshefedjian G, McMillan-Davey E, et al. Genetically decreased CYP2A6 and the risk of tobacco dependence: a prospective study of novice smokers. Tob Control 2004;13:422-8.

20. Cho MH, Castaldi PJ, Wan ES, Siedlinski M, Hersh CP, Demeo DL, et al. A genome-wide association study of COPD identifies a susceptibility locus on chromosome 19q13. Hum Mol Genet 2012;21:947-57.

21. Strasser AA, Malaiyandi V, Hoffmann E, Tyndale RF, Lerman C. An association of CYP2A6 genotype and smoking topography. Nicotine Tob Res 2007;9:511-8.

22. Pennanen M, Broms U, Korhonen T, Haukkala A, Partonen T, TuulioHenriksson A, et al. Smoking, nicotine dependence and nicotine intake by socio-economic status and marital status. Addict Behav 2014; 39:1145-51.

23. Mokdad AH, Marks JS, Stroup DF, Gerberding JL. Actual causes of death in the United States, 2000. JAMA 2004;291:1238-45.

24. Chiolero A, Faeh D, Paccaud F, Cornuz J. Consequences of smoking for body weight, body fat distribution, and insulin resistance. Am J Clin Nutr 2008;87:801-9.

25. Thorgeirsson TE, Gudbjartsson DF, Sulem P, Besenbacher S, Styrkarsdottir U, Thorleifsson G, et al. A common biological basis of obesity and nicotine addiction. Transl Psychiatry 2013;3:e308.

26. Kvaavik E, Meyer HE, Tverdal A. Food habits, physical activity and body mass index in relation to smoking status in 40-42 year old Norwegian women and men. Prev Med 2004;38:1-5.

27. Jimenez-Ruiz C, Miravitlles M, Sobradillo V, Gabriel R, Viejo JL, Masa JF, et al. Can cumulative tobacco consumption, FTND score, and carbon monoxide concentration in expired air be predictors of chronic obstructive pulmonary disease? Nicotine Tob Res 2004;6:649-53.

28. Shahab L, Jarvis MJ, Britton J, West R. Prevalence, diagnosis and relation to tobacco dependence of chronic obstructive pulmonary disease in a nationally representative population sample. Thorax 2006;61: 1043-7.

29. Bano R, Mahagaonkar AM, Kulkarni NB, Ahmad N, Nighute S. Study of pulmonary function tests among smokers and non-smokers in a rural area. Pravara Med Rev 2009;4:11-6.

30. Boskabady MH, Mahmoodinia M, Boskabady M, Heydari GR. Pulmonary function tests and respiratory symptoms among smokers in the city of Mashhad (north east of Iran). Rev Port Pneumol 2011;17:199204.

31. Boskabadi MH, Dehghani H, Esmaeilzadeh M. Pulmonary function tests and their reversibility in smokers. J Respir Dis Thorac Surg Intensive Care Tuberc 2003;2:23-30. 
Appendix 1. Multiple logistic regression analysis of the risk of obstructive ventilatory disturbance group according to the TTFC fully adjusted for cigarettes per day, smoking duration, age, income, education, and physical activity

\begin{tabular}{ccc}
\hline \multirow{2}{*}{ Predictor } & \multicolumn{2}{c}{ Outcome (obstructive pulmonary impairment) } \\
\cline { 2 - 3 } & Odds ratio (95\% confidence interval) & P-value \\
\hline TTFC (min) & & \\
$\leq 5$ & $7.338(1.967-27.366)$ & 0.003 \\
$6-30$ & $1.850(0.533-6.421)$ & 0.332 \\
$31-60$ & $1.820(0.586-5.657)$ & 0.300 \\
$>60$ & 1 & \\
\hline
\end{tabular}

TTFC, time to first cigarette. 